\title{
EDITORIAL The ethics of research publication
}

\author{
European Journal of Clinical Nutrition (2017) 71, 569; \\ doi:10.1038/ejen.2017.20
}

Faced with the exponential rise in published nutrition and biomedical research and an unbowed optimism of scientific writings, we are sometimes left with the feeling that major health issues have been already solved, or are at least going to be solved soon. Yet, each new paper opens up an unlimited number of further research questions that are immediately tackled by new research trials and their outcomes promptly communicated. This cycle of research enquiry and its dissemination augurs well for the discipline. In fact, during the last 20 years, we have had a flourishing era of science. However, it is worthwhile to remember that 20 years ago scientists had the same feeling!

Advances in research relate to increased new knowledge, and sometimes, medical breakthroughs. For this to be sustained, there must concomitantly be a substantial increase in governmental and industry funding, to allow for such research opportunities. Today, research is dominated by huge biomedical research bodies, national and international networks of scientists and institutions, as well as the industry. This is accompanied by the economization of science, where money now drives our institutions, scientists and research itself. Today many institutions and scientists have exponentially increased their research profits based on the number of publications. For example, Europe's leading research body, the French National Institute of Health and Medical Research (Inserm) now refers to about 12000 publications per year as their research gain. ${ }^{1}$

The primary motivation of medical research is that it will translate to a better and longer life for those who are ill in the population. However, biomedical research has also become a question of power, research dollars and a means to get ahead for scientists. Thus, for a scientist, publishing (or at least being an author in a long list of other authors) has become a precondition of their survival. One of us has previously indicated how libertarian principles (known from economics) determine the success and the future of biomedical research; high profit (that is, high scientific output) means everything. ${ }^{2}$ However, this high pressure carries a considerable risk for scientists, scientific journals and also for science itself. If publishing becomes a justification in its own right, it destroys the primary motivation for scientific enquiry.

To meet the need of scientists to publish, the number of new journals themselves have also exponentially increased, promising insanely quick turn around times and better coverage in numerous databases and social media; sometimes for a sizeable fee. There is considerable competition between traditional journals and newer open access formats, which impinges on journal survival too. Obviously there is pressure for both parties; the scientist and the publisher.

Recently it was brought to light that there is already a black market for scientific papers. ${ }^{3}$ There is now a list of 'papers for sale'. The better the journal and the higher you wish to be on the author list, the greater is the price of admission! Thus, becoming a co-author of an article to be published in a ranked journal depends on the money you pay. This kind of scientific misconduct has been already mentioned in $2014 .{ }^{4}$ It is up to our scientific community to keep an eye on what happens to science, and what we, as editors, need to continually do to keep science sacrosanct. While scientific misconduct may be related to the failure of individual scientists, it could also relate to systemic problems in research institutions and funding bodies.

Scientific journals have to maintain their high standard by recognizing and eliminating misconduct. Two former members of a scientific society's ethics committee developed an editorial process that lists seven warning signs that could be used to detect fraud, and these are: ${ }^{3}$

1. A cover letter that is substantially worse in grammar, spelling and writing quality than the accompanying manuscript.

2. Few shared co-authored papers between combinations of authors.

3. Few authored papers for individual authors.

4. Few to no citations of papers by individual co-authors in the manuscript bibliography.

5. An absence of previous publications by one or more co-authors in the field of the manuscript.

6. The same e-mail address used for multiple authors.

7. Textual overlap with other papers.

These are good suggestions as they flag potentially suspect submissions to journals. However, as acknowledged by these authors, such criteria need to evolve as unethical scientists will find a way.

In conclusion, the high pressures of modern day science drives the success of medical research, but may also come at a cost to scientific quality, the scientist and centres of higher learning, as well as the scientific journals. Some scientists choose to walk the tight-rope. As editors, we take the stand that all forms of scientific misconduct are due to the individual researcher. While naming and shaming them is the immediate fallout, long-term bans from publishing would be difficult to police, given the significant rise in predatory journals. ${ }^{5}$ In some way, misconduct in science also relates to those systems of funding that award more research dollars to those who provide more 'bang' for the initial investment. Measuring success as numbers of papers, rather than the quality of the science and its impact, is also to blame. There is an urgent need for our scientific community to engage in fruitful discussions on these issues, and this editorial is the first step in that direction.

\section{CONFLICT OF INTEREST}

The authors declare no conflict of interest.

MJ Müller ${ }^{1}$ and M Soares ${ }^{2}$ ${ }^{1}$ Department of Human Nutrition and Food Science, Christian-Albrechts-University of Kiel, Kiel, Germany and

${ }^{2}$ School of Public Health, Curtin University, Perth, WA, Australia E-mail: mmueller@nutrfoodsc.uni-kiel.de

\section{REFERENCES}

1 Casassus B. Research focus. Profile: Inserm, Paris, France. Lancet 2016; 388: 2973.

2 Müller MJ, Landsberg B, Ried J. Fraud in science: a plea for a new culture in research. Eur J Clin Nutr 2014; 68: 411-415.

3 NN. 7 signs a scientific paper's authorship was bought. Available at: http:/retractionwatch.com/2016/10/24/seven-signs-a-paper-was-for-sale/\#respond (accessed on 24 January 2016).

4 Seife C. For sale: 'Your name here' in a prestigious science journal. Sci Am 2014; e-pub ahead of print 17 December 2014.

5 Publons. Beall's list: gone but not lost. Available at: https://publons.com/blog/beallslist-gone-but-not-lost/?utm_source $=$ PublonsUsers\&utm_campaign $=8 \mathrm{e} 0 \mathrm{e} 613 \mathrm{~d} 95$ Mixed_reviews_issue_7_AB\&utm_medium $=$ email\&utm_term $=0$ _d203ec3f11-8e0e 613d95-136291653 (accessed on 24 January 2016). 